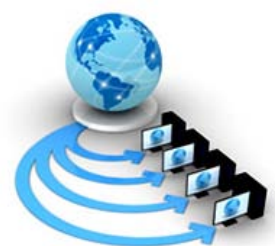

Volume 8, No. 7, July - August 2017

International Journal of Advanced Research in Computer Science

RESEARCH PAPER

\title{
CLASSIFICATION TECHNIQUE FOR DRUG DISCOVERY IN MEDICAL IMAGE PROCESSING
}

\author{
Neha Tandon \\ Research Scholar \\ Computer Science \& Engineering Department, \\ I.K.G.P.T.U., Kapurthala, Punjab.
}

\author{
Pankaj Bhambri \\ Assistant Professor \\ Information Technology Department, \\ Guru Nanak Dev Engineering College, Ludhiana, Punjab.
}

\begin{abstract}
A complex structure that focuses on the analysis of variety of levels in regular manner is known as the ontology process. There has been an involvement in the programmed learning methods for constructing the ontology which is completely a level-based method. There are different strategies included for various levels. There are all these levels that are defined at various levels. For the purpose of mapping the two base classes, the object properties are utilized. In this work, technique is been proposed which is based on the neural networks which will mark the area for which the drug need to be discovered. The proposed technique is implemented in MATLAB and it is been analyzed that accuracy is increased and execution time is reduced.
\end{abstract}

\section{INTRODUCTION}

The types, properties and the interrelationships of the objects that are truly present within the specific domain of discourse are named formally within the ontology. The capacity to evaluate the specific approach is the main objective here. The similar ideas are to be considered here within this approach. During the management of the abstractions within the ontologies, there is a same process followed by the semantic web explore area. For the purpose of conceptualizing knowledge, the ontologies provide a specific data structure [1]. By involving completely different knowledge related to these concepts, new methods are to be generated here. There are two categorizations of the phylogentic methods are distance based method and character based method. The evolutionary information that is known is used for the character based methods [2]. On the basis of the distance matrix, the cluster based computations are gathered. There are two steps involved here. The distance based clustering method is referred to as the Neighboring Joining Method. The collection of each of the gathered species is evaluated and further, for the resulting tree, the sum of length of the branch is computed. The collection of two species that have least distance amongst them as per the distance matrix is known as the Unweighted Pair Group Method utilizing Arithmetic Average (UPGMA) [3]. The character based method that is utilized for handling the phylogeny relationships from the various organisms is known as the maximum parsimony method. A prevalent factual method that is utilized for fitting the measureable model to the data is known as maximum probability estimation (MLE). For the factual inferences, this method is mainly involved. From the set of sequences, the phylogenetic tree is allowed to be worked by the pipeline within the core system. A succession of the Perl modules that wrap the distinctive external programming programs within this pipeline mainly requires three modes [4]. Various other programs are also required here that are not mentioned here which are required for making the junction between the main programs, providing output to the results within various formats, and modifying the tree or rearranging it to provide various editions within it.

On the basis of properties of quantum mechanics the simulating molecules are allowed to interact with each other within the structural bioinformatics tools. This has been developed with the advancements in the biomedical research area. The techniques developed have been a part of the drug design and drug discovery methods. The gathered information is used for identification, design and optimization of newly evolving drugs within this field [5]. The discovery of a molecule that is ready to bind and activate or inhibit a molecular target which is mainly a protein, is done in the drug search and discovery method. A GPU is a particular device designed to rapidly manipulate high measures of graphical pixels. Historically, GPU were conceived for being used in advanced graphics and videogames. All the more as of late interfaces have been worked to interact with codes not identified with graphical purposes, for instance for linear algebraic controls [6]. The involvement of GPU for providing basic reason logical and engineering computing is known as the general-reason GPU computing method.

KUDA approach: The overhead of the customary method is minimized with the help of division of monitoring and analyzing of CPU and GPU together. Here, both of the tasks are executed on the similar threads or cores. The speed of program and the examination code is similar. Once the program terminates, the code also ends soon after that. The implementation of a prototype tool known as KUDA is done here. The KUDA is linked with the collection of multithreaded benchmarks. There are two parts in which the KUDA algorithm is divided [7]. The first part involves the core functionality which includes the routines in which the events are recorded, the event frames are manages and the execution of race detection kernels on the GPU, all within the dynamic library. The x86 binaries are to be dynamically instrumented with the help of Pin tool in the secondary part [8]. The main objective here is to callback the routines within the dynamic library in respect of specific events. The multithreaded programs that are created with the help of pthreads library are supported by the Pin tool. 


\section{LITERATURE REVIEW}

Vatanjeet Singh(2016) proposed in this paper [9] an ultra wide band (UWB) method which is a multi resonant optical antenna which involves the FR4 dielectric constant 4.4 within it. Within this proposed antenna design the gap coupled feeding system is also involved. There is copper material utilized within the ground, fix as well as the feed line of the system. The proposed antenna covers diverse range of applications, for example, detection of explosives, drugs (cocaine, heroin), colon cancer, mind tumor and security applications, for example, mail screening examination and monitoring water content in leaves.

Nima Aliakbarinodehi(2015) proposed in this paper [10] a proper survey proposed on the nano-structure biosensors with respect of the electro-active cancer-drug identification. The enhancement of level of sensitivity and the limit of detection of the two different nano-structured biosensors is focused in this paper. There are numerous other factors as well that effect the impact of dimensions of nano-particles on the performance and the material science involved here. This is the main highlight of the future studies to be presented ahead.

(1) Patricia Vazquezet.al, (2014) proposed in this paper [11] the integration of an electrochemical miniaturized scale within a hollow microneedle. The recognition of the concentration of drugs present within the fluids of body is the main objective of this new sensor platform being derived in this paper. It is seen through the various calibrations provided for the sensitivity of the systems; the various experiments have been conducted. These results are also compared with the already existing highly-sensitive techniques. As per the simulation results achieved, there have been depicted huge enhancements in the results and these enhancements have resulted in enhancing the systems on the basis of various aspects.

(2) Pedro R. Gomes, et.al, (2011) provided in this paper [12] a controlled drug administration for the patients that are suffering from a particular type of disease known as Brugada Syndrome (BS). Under the proper electrocardiogram (ECG) monitoring, there are various types of drugs that are provided under proper surveillance and the quantity provided is also monitored. There have been various enhancements proposed within the paper that have increased the efficiency of the existing mechanisms and the methods have been involved within the trending applications to provide accurate results. The results are compared with the results achieved from existing approaches also.

Daniela De Venuto(2011) proposed in this paper [13], an electrochemical biosensor for the molecules that are involved within the personalized machine. The type of drug is differentiated by the potential systems and the concerned systems also provide the amount of drug concentrated there. In this paper, an example of variations is proposed in this paper along with a new design that might help in multiplexing the biosensing across various $\mathrm{pH}$ and temperature control systems. For the need of monitoring the personal therapies of the patients, the systems proposed and designed within this paper are ensured to be reliable and robust so that they prove to be beneficial to the current applications.
S. Sara Ghoreishizadeh(2011) proposed in this paper [14] an anti-cancer drug known as Etoposide has been selected as model for cyclic voltammetry drug identifications. The sensitivity of the system is enhanced with the involvement of carbon nanotubes that are the electron-exchange mediators. The devices that are completely implantable and remotely powered within certain applications are to be considered here as there is very less power consumption within such services. There will be a fabrication of current readout circuit on a chip for providing drug detection that is based on the $\mathrm{CV}$ mechanism.

\section{RESEARCH METHODOLOGY}

GPU becomes a highly parallel processor for the purpose of heavy workloads due to its ease of being programmed by Compute Unified Device Architecture (CUDA). GPU is an array which contains number of streaming processors (SPs). It also includes Single Instruction Multiple Threads multiprocessors. Memory spaces are also available in the GPU which is helpful to microprocessors. A thread is known as the basic execution unit of a CUDA programming model. The GPU architecture is based to discovery new patterns for the drug discovery. To increase the accuracy of drug discovery technique c-mean clustering is applied with the SVM classifier.

\section{Pseudo Code of Proposed Algorithm}

Step 1: Input the training and test data for the drug classification

Step 2: Repeat while loop for clustering

Step 2.1: Start for loop to input training set data

Step 2.1.1: Apply c-mean technique to clustering of the data End for

End While

Step 3: Repeat while unless all the data get classified

Step 3.1: Match the training data and test data features using SVM classifier

Step 3.2: Classify most similar and dissimilar data

End while 


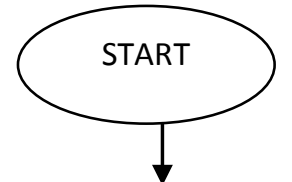

Input the image on which similarity and dissimilarity need to be calculated

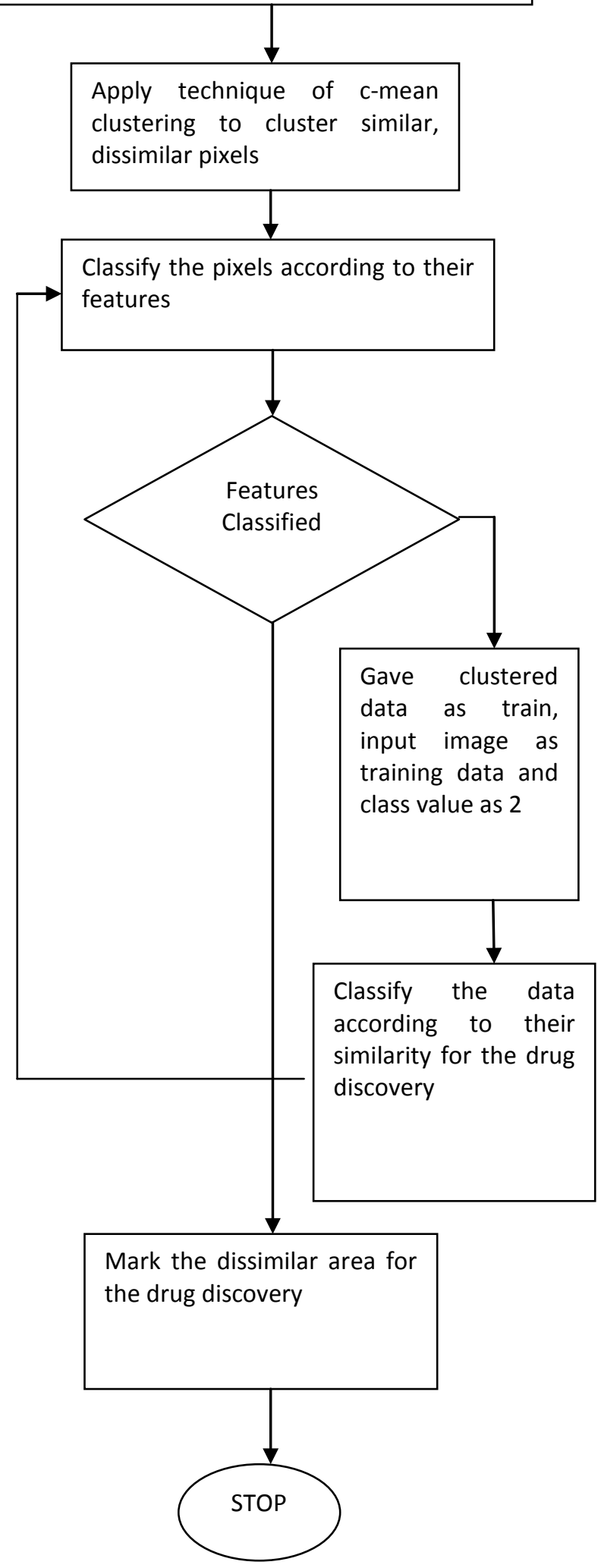

Fig 1: Proposed Flowchart

\section{EXPERIMENTAL RESULTS}

\section{PSNR Comparision}

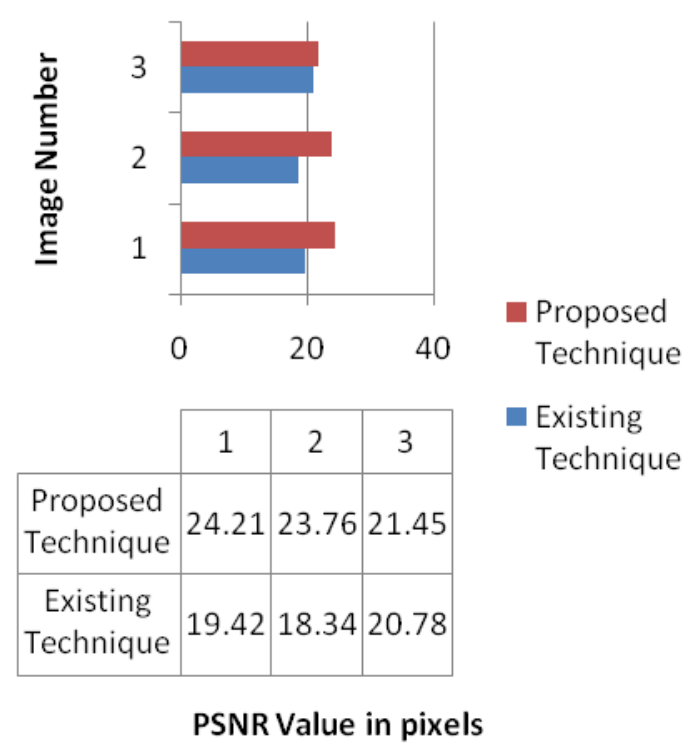

Fig 2: PSNR Comparison

As shown in the figure 2, The PSNR comparison of proposed and existing algorithm is done. It is been analyzed that PSNR of the proposed algorithm is more as compared to existing due to the use of classification technique

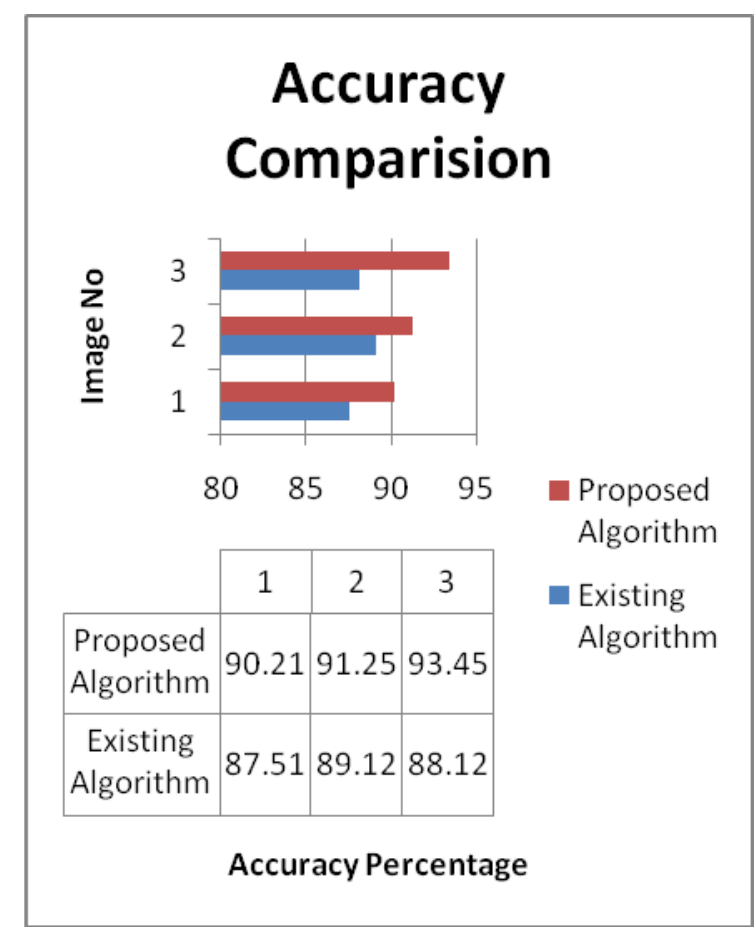

Fig 3: Accuracy Comparison

As shown in the figure 3, the accuracy of the proposed and existing technique is compared and it is been analyzed that due to use of classification technique accuracy is increased at steady rate. 


\section{CONCLUSION}

For the purpose of lifting the performance of GPU in terms of its high-performance, the speed of the application is made to be higher in terms of the view of client. The code will keep running in a highly parallel manner on the GPU. Further, a parallel code can be written on the basis of this which can help in scaling the devices which have various parallel processing capabilities. The memory modules that can be physically separated from each other can be executed on the basis of CPU and GPU. In this work, it is been concluded that classification technique is applied which will classify the area for which disease need to discovered. The proposed technique is implemented in MATLAB and analyzed that accuracy is increased and execution time is reduced.

\section{REFERENCES}

[1] Buneman P., "Mathematics in the Archaelogical and Historical Sciences”, 1971, F Hodson Edinburgh Univ Press, Edinburgh, UK, pp 387-395.

[2] Gascuel O, Steel M, "Neighbor-joining revealed”, 2006, Mol Biol Evol 23:1997-2000.

[3] Aitken AC, "On least squares and linear combinations of observations”, 1935, Proc R Soc Edinburgh A 55:42-48

[4] Vinh S, von Haeseler, "A Shortest triplet clustering: Reconstructing large phylogenies using representative sets", 2005, BMC Bioinf 6:92

[5] Jukes T, Cantor C, "Mammalian Protein Metabolism”, 1969, H Munro (Academic, Waltham, MA), pp 21-132.
[6] Beyer W, Stein M, Smith T, Ulam S, “A molecular sequence metric and evolutionary trees”, 1974, Math Biosci 19:9-25

[7] Atteson K, "The performance of neighbor-joining methods of phylogenetic reconstruction”, 1999, Algorithmica 25:251-278

[8] Susko E, Inagaki Y, Roger A, "On inconsistency of the neighbor-joining, least squares, and minimum evolution estimation when substitution processes are incorrectly modeled”, 2004, Mol Biol Evol 21:1629-1642

[9] Vatanjeet Singh, Aman Nag, Ekambir Sidhu,” High Gain Ultra Wide Band (UWB) Multi Resonant Antenna for Biomedical Applications, Security Purposes and Drug Detection”, 2016, IEEE, 978-1-4673-9338-6

[10]Nima Aliakbarinodehi, Giovanni De Micheli, Sandro Carrara," Optimized Electrochemical Detection of AntiCancer Drug by Carbon Nanotubes or Gold Nanoparticles", 2015, IEEE, 978-1-4799-8229-5

11] Patricia Vazquez, Conor O’Mahony, Joseph O’Brien, Grégoire Herzog,” Microneedle sensor for voltammetric drug detection in physiological fluids", 2014, IEEE, 978-1-47990162-3

[12] Pedro R. Gomes, C. S. Lima, Filomena O. Soares, J. H. Correia," Automatic Continuous ECG Monitoring System for Over-drug Detection in Brugada Syndrome”, 2011, IEEE, 978-1-4244-4122-8

[13] Daniela De Venuto, Sandro Carrara, Andrea Cavallini, Giovanni De Micheli,” pH Sensing with Temperature Compensation in a Molecular Biosensor for Drugs Detection”, 2011, IEEE, 978-1-61284-914-0

[14] S. Sara Ghoreishizadeh, Camilla Baj-Rossi, Sandro Carrara, Giovanni De Micheli”, Nano-Sensor and Circuit Design for Anti-Cancer Drug Detection”, 2011, IEEE, 978-1-4577-04222 